\title{
Análisis de riesgo en limpieza de productos semisólidos en el laboratorio farmacéutico de la Dirección Nacional de Sanidad de las Fuerzas Armadas
}

\section{Risk analysis in cleaning semi-solid products in Armed Forces National Health Direction pharmaceutical laboratory}

doi) http:// dx.doi.org/10.35954/SM2019.38.1.2
Jeannette Araújo a (D) https://orcid.org/0000-0002-3299-2791
Martín Daners a (D) https://orcid.org/0000-0002-0213-9271
Mariela Falero a (D) https://orcid.org/0000-0001-9720-4058
Sabrina Laulhé a (1D https://orcid.org/0000-0002-7848-678X
Mariana Poggi a (D) https://orcid.org/0000-0003-4282-6102

(a) Laboratorio Farmacéutico de la Dirección Nacional de Sanidad de las Fuerzas Armadas. Montevideo. Uruguay.

\section{RESUMEN}

La validación de limpieza es una herramienta clave para asegurar la calidad del producto final según las Buenas Prácticas de Fabricación. Permite detectar riesgos de contaminación y contaminación cruzada. En este trabajo, mediante el estudio de riesgo de los 18 productos semisólidos fabricados utilizando 21 principios activos diferentes, se seleccionan los activos críticos empleados y se calcula el límite aceptable superficial de los mismos. La unidad posológica de un producto semisólido no está claramente definida como en comprimidos. En este estudio se utiliza la unidad fingertip unit (FTU), conociendo la cantidad de activo contenido en una FTU y la cantidad de FTU necesaria para cubrir la zona afectada, se determina la cantidad de activo por aplicación y se calcula el límite. Los activos críticos seleccionados fueron Salicilato de octilo, Límite $=0.0690 \mathrm{mg} /$ $\mathrm{mL}$ y Nitroglicerina, Límite $=0.0002 \mathrm{mg} / \mathrm{mL}$.

PALABRAS CLAVE: Contaminación; Estudios de Validación; Laboratorios; Medición de Riesgo.

\begin{abstract}
The validation of cleaning is a key tool to ensure the quality of the final product, according to Good Manufacturing Practices. It allows to detect contamination risks and cross contamination. In this paper, by means of the risk study of the 18 semisolid products manufactured using 21 different active principles, critical active ingredients used are selected and the acceptable superficial limit of them is calculated. The dosage unit of a semisolid product is not clearly defined as in tablets. In this study, the fingertip unit (FTU) is used, knowing the amount of active ingredients contained in an FTU and the amount of FTU needed to cover the affected area, the amount of active ingredient per application is determined and the limit is calculated. The critical active ingredients selected were octyl salicylate, Limit $=0.0690 \mathrm{mg} / \mathrm{mL}$ and Nitroglycerin, Limit $=0.0002 \mathrm{mg} / \mathrm{mL}$.
\end{abstract}

KEY WORDS: Contamination; Validation Studies; Laboratories; Risk Assessment. 


\section{INTRODUCCIÓN}

La elaboración de productos farmacéuticos se rige por las Buenas Prácticas de Manufactura, GMP en inglés. Estas normas constituyen estándares internacionales de calidad, adoptados en nuestro país por la autoridad sanitaria, que aseguran que el producto farmacéutico cumple con los requisitos de calidad y seguridad correspondientes.

Uno de los aspectos incluidos en las Buenas Prácticas de Manufactura, consiste en garantizar la limpieza de los equipos utilizados en la elaboración así como también, prevenir la contaminación cruzada entre los múltiples productos que se fabrican en un mismo equipo. Esto se logra mediante la validación de los procesos de limpieza. La validación consiste en la generación de evidencia documentada que demuestre que un procedimiento de limpieza es consistentemente capaz de limpiar a predeterminados niveles de limpieza.

El alcance de este trabajo se centra en la determinación de los activos críticos utilizados en formas farmacéuticas semisólidas y el establecimiento de los límites de residuo en superficie, resolviendo los problemas que surgen a la hora de dicha determinación por la propia forma farmacéutica.

La forma farmacéutica semisólida se refiere a especialidades farmacéuticas o cosméticas que se aplican sobre la piel y mucosas.

La dificultad para el cálculo de las dosis en formulaciones tópicas se debe a que carecen de unidades posológicas definidas y a que la zona de aplicación es variable según el área corporal afectada. Se utiliza como unidad posológica fingertip unit (FTU). Es una medida estándar utilizada en productos semisólidos (cremas, ungüentos, geles) que permite conocer la cantidad de producto contenida en cada aplicación y por tanto la cantidad de activo. Por definición una FTU equivale a $500 \mathrm{mg}$ de producto (1). Los activos seleccionados y límites establecidos serán usados en una instancia posterior como marcadores durante la ejecución de la validación de limpieza en dicho sector.

\section{OBJETIVO}

El objetivo de este trabajo es seleccionar los activos críticos empleados en la fabricación de semisólidos y calcular el límite superficial de los mismos.

\section{MATERIALES}

Planilla electrónica de procesamiento de datos. Procedimientos de limpieza del sector semisólidos.

\section{METODOLOGÍA}

Para la selección de los activos críticos se utilizó el criterio del peor caso, en inglés worst case. Consiste en seleccionar entre todos los principios activos de los productos fabricados, el/los más peligroso/s. Son considerados los más peligrosos aquellos más tóxicos y difíciles de limpiar. Se agruparon los productos según los equipos que comparten, en dos trenes de equipos, uno de ellos utilizado para lotes grandes (mayores a $15 \mathrm{Kg}$ de producto) y otro tren para lotes pequeños (menores de $10 \mathrm{Kg}$ de producto).

\section{1- Selección de activos a validar}

Se realizó un estudio de riesgo basado en el análisis del peor caso, para seleccionar el activo a validar. Se considera el caso de un producto contaminado con el activo más peligroso y que de presentar contaminación expondría de forma más importante al consumidor final.

Se seleccionaron parámetros claves para determinar la peligrosidad de los activos y se asignaron puntajes a los mismos para confeccionar la matriz del estudio de riesgo. Las propiedades seleccionadas y los puntajes asignados se representan en la figura 1.

\section{2- Cálculo de límites}

Una vez seleccionado el activo a validar, se establece la cantidad máxima del mismo que puede permanecer sobre la superficie del equipo luego de aplicar el procedimiento de limpieza, de manera que no presente riesgo de contaminación sobre el siguiente producto. Dicha cantidad es el límite de limpieza.

Para el cálculo del límite se adopta el método desarrollado por Fourman y Mullen de la compañía Eli Lilly aplicado en este caso a semisólidos (2). Dicho 


\begin{tabular}{|c|c|c|c|}
\hline & Descripción & Puntaje \\
\hline & & Muy soluble & 1 \\
\hline \multirow{7}{*}{\multicolumn{2}{|c|}{$\begin{array}{l}\text { Solubilidad en agua: la mayor } \\
\text { parte de la limpieza se realiza con } \\
\text { agua o con soluciones acuo- } \\
\text { sas. Un activo poco soluble en } \\
\text { agua lógicamente tendrá menos } \\
\text { probabilidades de ser removido } \\
\text { exitosamente en la limpieza. }\end{array}$}} & Libremente soluble & 1 \\
\hline & & Soluble & 2 \\
\hline & & Moderadamente soluble & 2 \\
\hline & & Levemente soluble & 3 \\
\hline & & Muy levemente soluble & 3 \\
\hline & & Prácticamente insoluble & 3 \\
\hline & & Insoluble & 3 \\
\hline \multirow{4}{*}{\multicolumn{2}{|c|}{$\begin{array}{l}\text { Dificultad de limpieza: se asigna } \\
\text { el siguiente puntaje según la } \\
\text { dificultad de limpieza de cada } \\
\text { especialidad farmacéutica. }\end{array}$}} & Dificultad de limpieza & Puntaje \\
\hline & & Geles & 1 \\
\hline & & Cremas & 2 \\
\hline & & Ungüentos & 3 \\
\hline \multirow{7}{*}{$\begin{array}{l}\text { Toxicidad: deter- } \\
\text { minada mediante } \\
\text { la LD50 en ratas, } \\
\text { por vía oral, está } \\
\text { directamente } \\
\text { relacionada con } \\
\text { la peligrosidad del } \\
\text { activo. }\end{array}$} & LD50 $(\mathrm{mg} / \mathrm{Kg})$ & Descripción & Puntaje \\
\hline & 15000 & Prácticamente no tóxico & 1 \\
\hline & $5000-14999$ & Levemente tóxico & 1 \\
\hline & $500-4999$ & Moderadamente tóxico & 2 \\
\hline & $50-499$ & Muy tóxico & 3 \\
\hline & $5-49$ & Extremadamente tóxico & 4 \\
\hline & $<5$ & Súper tóxico & 5 \\
\hline
\end{tabular}

Figura 1. Matriz del estudio de riesgo.

método establece que existen tres criterios para el cálculo del límite de limpieza. Se debe calcular el límite según cada criterio y tomar el menor, es decir, el más exigente o peor caso (worst case). Los tres criterios de cálculo son:

\section{1- Criterio de dosis}

Un activo A puede estar presente como contaminante en el producto siguiente fabricado $B$, en una milésima parte de su dosis mínima diaria:

$\mathrm{L}_{1}(\mathrm{mg} / \mathrm{mL})=\mathrm{FS} \times \mathrm{D}_{\text {minA }} \times$ TLB $\times \mathrm{a}_{\mathrm{h}} / \mathrm{D}_{\text {máxprodB }} \times \mathrm{a}_{\mathrm{c}} \times \mathrm{V}_{\text {des }}$ $\mathbf{D}_{\text {minA }}$ : dosis mínima diaria de activo $A(m g), \mathbf{D}_{\text {máxprodB }}$ : dosis máxima diaria de producto $B(\mathrm{mg}), \mathbf{F S}$ : factor de seguridad (0.001), TLB: tamaño de lote de $B(\mathrm{mg}), \mathbf{a}_{\mathrm{h}}$ : área hisopada $\left(\mathrm{cm}^{2}\right), \mathbf{a}_{\mathbf{c}}$ : área del tren $\left(\mathrm{cm}^{2}\right), \mathbf{V}_{\text {des }}$ : volumen de desorción del hisopo $(\mathrm{mL}), 5 \mathrm{~mL}$ en todos los casos.

Cuando se trata de productos tópicos el cálculo de las dosis mínimas o máximas diarias se dificulta ya que no se encuentran divididas en unidades individuales de dosificación, a diferencia de las formulaciones de sólidos y líquidos. Sumado a esto, el tamaño de dosis por aplicación varía dependiendo del área total de la lesión.
Por lo tanto, surge la necesidad de establecer un método racional para determinar los límites de limpieza.

El criterio establecido por Long y Finley (2) describe la dosis de productos tópicos en términos de unidades de la punta del dedo (FTUs por Fingertip units). Una FTU de adulto es la cantidad de semisólido expresada desde un tubo con un diámetro estándar de boquilla de $5 \mathrm{~mm}$ de diámetro, aplicado desde el pliegue distal a la punta del dedo índice. Contiene aproximadamente $0.5 \mathrm{~g}$ de crema y se asume que aproximadamente 20.25 g ( 40.5 FTUs) de crema se necesitan para cubrir un cuerpo adulto. Según los autores 1 FTU abarca alrededor de 286 $\mathrm{cm}^{2}$ de piel. Esto implica que aproximadamente $1,75 \mathrm{mg}$ de producto se aplica por $\mathrm{cm} 2$ de piel. De acuerdo a esto se calculan las dosis como sigue:

\section{$D_{\text {minA }}=\% A \times 1.75 \mathrm{mg} / \mathrm{cm}^{2} \times$ área de aplicación}

$\left(\mathrm{cm}^{2}\right) \times$ aplicaciones de $A$ al día / 100

$D_{\text {máxB }}=1.75 \mathrm{mg} / \mathrm{cm}^{2} x$ área de aplicación $B\left(\mathrm{~cm}^{2}\right) \times$ aplicaciones de $\mathrm{B}$ al día

Para determinar el área afectada se considera la indicación de uso del producto y la equivalencia:

$1 \mathrm{FTU}=286 \mathrm{~cm}^{2}$ (3) (figura 2).

\section{2- Límite por defecto}

El valor máximo de $A$ que puede presentarse en B es 10 ppm:

$\mathrm{L}_{2}(\mathrm{mg} / \mathrm{mL})=10 \mathrm{ppm} \times \mathrm{TL}_{\text {prodB }} \times \mathrm{a}_{\mathrm{h}} / \mathrm{a}_{\mathrm{c}} \times \mathrm{V}_{\text {des }}$

$\mathrm{TL}_{\text {prodB }}$ : tamaño de lote $\mathrm{B}(\mathrm{Kg}, \mathrm{L})$

\section{3- Límite visual}

Es un valor fijo $0.1 \mathrm{mg} / 25 \mathrm{~cm}^{2}$.

\section{RESULTADOS}

Para el tren de equipos utilizado en lotes grandes, se valoraron los siguientes productos, se determinó el producto de mayor riesgo y se calcularon los límites de limpieza. Los resultados se presentan en las figuras 3,4 y 5 . 


\begin{tabular}{|c|c|c|}
\hline Zona a aplicar & N FTU & $\begin{array}{c}\text { Área de } \\
\text { aplicación }\left(\mathbf{c m}^{2} \mathbf{)}\right.\end{array}$ \\
\hline Todo el cuerpo & 40,5 & 11583 \\
\hline Zona perianal & 1 & 286 \\
\hline Zona del pañal en bebés & 3,5 & 1001 \\
\hline Manos & 1 & 286 \\
\hline Abdomen & 3,5 & 1001 \\
\hline
\end{tabular}

Figura 2. Número de FTUs a aplicar según zona afectada.
Para lotes grandes el activo de mayor riesgo es salicilato de octilo, cuyo límite es $\mathrm{L}_{2}=0.0690 \mathrm{mg} / \mathrm{ml}$. Para tren de equipos utilizado en lotes chicos se determinó el producto de mayor riesgo y se calcularon los límites de limpieza. Los resultados se presentan en las figuras 6,7 y 8 .

Para tren de equipos utilizado en lotes chicos el activo de mayor riesgo es nitroglicerina, cuyo límite es $\mathrm{L}_{1}=0.0002 \mathrm{mg} / \mathrm{ml}$.

\begin{tabular}{|c|c|c|c|c|c|c|c|c|c|}
\hline \multicolumn{10}{|c|}{ Selección del producto crítico, A. Semisólidos. Lotes grandes. } \\
\hline Producto & Activo & Tipo & Solubilidad en agua & Valor & $\begin{array}{l}\text { LD50 } \\
\text { (mg/kg) }\end{array}$ & Toxicidad & Valor & $\begin{array}{c}\text { Dificultad } \\
\text { de limpieza }\end{array}$ & $\begin{array}{l}\text { RIESGO } \\
\text { TOTAL }\end{array}$ \\
\hline Protector solar & $\begin{array}{l}\text { Silicato de octilo } \\
\text { (Escalol 587) }\end{array}$ & Crema & insoluble & 3 & 200 & muy tóxico & 3 & 2 & 8 \\
\hline Secavit & Vitamina A & Ungüento & insoluble & 3 & 6060 & levemente tóxico & 1 & 3 & 7 \\
\hline Salisán & Salicilato de metilo & Crema & levemente soluble & 3 & 887 & moderadamente tóxico & 2 & 2 & 7 \\
\hline Salisán & Alcanfor & Crema & prácticamente insoluble & 3 & 1310 & moderadamente tóxico & 2 & 2 & 7 \\
\hline Ungüento vitaminado & Vitamina A & Ungüento & insoluble & 3 & 6060 & levemente tóxico & 1 & 3 & 7 \\
\hline Argiderm & Clorfeniramina & Crema & soluble & 2 & 306 & muy tóxico & 3 & 2 & 7 \\
\hline Argiderm & Mentol & Crema & insoluble & 3 & 2900 & moderadamente tóxico & 2 & 2 & 7 \\
\hline Saniproct & Hidrocortisona base & Ungüento & muy levemente soluble & 3 & 5000 & levemente tóxico & 1 & 3 & 7 \\
\hline Saniproct & Lidocaína clorhidrato & Ungüento & muy soluble & 1 & 292 & muy tóxico & 3 & 3 & 7 \\
\hline Saniproct & Tetracaina clorhidrato & Ungüento & muy soluble & 1 & 160 & muy tóxico & 3 & 3 & 7 \\
\hline Saniproct & Subgalato de bismuto & Ungüento & insoluble & 3 & 6370 & levemente tóxico & 1 & 3 & 7 \\
\hline Clotrimazol & Clotrimazol & Crema & prácticamente insoluble & 3 & 708 & moderadamente tóxico & 2 & 2 & 7 \\
\hline Clobetasol & Clobetasol propionato & Crema & prácticamente insoluble & 3 & 4000 & moderadamente tóxico & 2 & 2 & 7 \\
\hline Argiderm & Hidrocortisona base & Crema & muy levemente soluble & 3 & 5000 & levemente tóxico & 1 & 2 & 6 \\
\hline Argiderm & Lidocaina clorhidrato & Crema & muy soluble & 1 & 292 & muy tóxico & 3 & 2 & 6 \\
\hline Protector solar & $\begin{array}{c}\text { Benzofenona } 3 \\
\text { (Escalol 567) }\end{array}$ & Crema & insoluble & 3 & 7400 & levemente tóxico & 1 & 2 & 6 \\
\hline Protector solar & $\begin{array}{l}\text { p-metoxicinamato de } \\
\text { octilo (Escalol 557) }\end{array}$ & Crema & insoluble & 3 & 8000 & levemente tóxico & 1 & 2 & 6 \\
\hline Cortibiot & Hidrocortisona base & Gel & muy levemente soluble & 3 & 5000 & levemente tóxico & 1 & 1 & 5 \\
\hline Corticoide gel & Hidrocortisona base & Gel & muy levemente soluble & 3 & 5000 & levemente tóxico & 1 & 1 & 5 \\
\hline Vaselina sólida & Vaselina & Gel & insoluble & 3 & NA & NA & 0 & 1 & 4 \\
\hline Cortibiot & Neomicina sulfato & Gel & muy soluble & 1 & $>8000$ & levemente tóxico & 1 & 1 & 3 \\
\hline Gel alcohol $70^{\circ}$ & Etanol $95^{\circ}$ & Gel & soluble & 1 & 7060 & levemente tóxico & 1 & 1 & 3 \\
\hline Base hidrofilica & NA & Crema & NA & 0 & NA & NA & 0 & 2 & 2 \\
\hline Crema hidratante & NA & Crema & NA & 0 & NA & NA & 0 & 2 & 2 \\
\hline Gel para imagenología & NA & Gel & NA & 0 & NA & NA & 0 & 1 & 1 \\
\hline
\end{tabular}

Figura 3. Valoración de productos semisólidos en tren de equipos para lotes grandes.

\begin{tabular}{|c|c|c|c|c|c|c|c|}
\hline Producto & Activo & Tipo & Solubilidad en agua & Valor & $\begin{array}{c}\text { LD50 } \\
\text { (mg/kg) }\end{array}$ & Toxicidad & Valor \\
\hline Protector solar & $\begin{array}{c}\text { Silicato de octilo } \\
\text { (Escalol 587) }\end{array}$ & Crema & insoluble & 3 & 200 & $\begin{array}{c}\text { RIESGO } \\
\text { TOTAL }\end{array}$ \\
\hline
\end{tabular}

Figura 4. Producto con mayor riesgo total. 
Selección del producto B y cálculo del límite de limpieza.

\begin{tabular}{|c|c|}
\hline \multicolumn{2}{|c|}{ Producto A: salicilato de octilo } \\
\hline$\%$ A en FF & 8 \\
\hline FS & 0,001 \\
\hline área hisopada $\left(\mathbf{c m}^{2}\right)$ & 100 \\
\hline sup. compartida $\left(\mathbf{c m}^{2}\right)$ & 37670 \\
\hline
\end{tabular}

\begin{tabular}{|c|c|c|c|c|c|c|c|c|c|}
\hline Producto B & Activo B & Área de aplicación & $\begin{array}{c}\mathbf{g} \\
\text { FTU/aplicación }\end{array}$ & $\begin{array}{l}\text { Lote B } \\
(\mathrm{mg})\end{array}$ & $\begin{array}{c}\text { máx } \\
\text { ap/día }\end{array}$ & L1 (mg) & L2 (mg) & L3 (mg) & Observaciones \\
\hline Argiderm & Hidrocortisona & todo el cuerpo & 20,25 & 18000000 & 3 & 3,823 & 0,4778 & 0,4000 & \\
\hline Argiderm & Clorfeniramina & todo el cuerpo & 20,25 & 18000000 & 3 & 3,823 & 0,4778 & 0,4000 & \\
\hline Argiderm & Lidocaína clorhidrato & todo el cuerpo & 20,25 & 18000000 & 3 & 3,823 & 0,4778 & 0,4000 & \\
\hline Argiderm & Mentol & todo el cuerpo & 20,25 & 18000000 & 3 & 3,823 & 0,4778 & 0,4000 & \\
\hline Saniproct & Hidrocortisona base & zona rectal & 0,5 & 20000000 & 2 & 4,247 & 0,5309 & 0,4000 & $1 \mathrm{FTU}(1 \mathrm{FTU}=0,5 \mathrm{~g})$ \\
\hline Saniproct & Lidocaína clorhidrato & zona rectal & 0,5 & 20000000 & 2 & 4,247 & 0,5309 & 0,4000 & $1 \mathrm{FTU}(1 \mathrm{FTU}=0,5 \mathrm{~g})$ \\
\hline Saniproct & Tetracaína clorhidrato & zona rectal & 0,5 & 20000000 & 2 & 4,247 & 0,5309 & 0,4000 & $1 \mathrm{FTU}(1 \mathrm{FTU}=0,5 \mathrm{~g})$ \\
\hline Saniproct & Subgalato de bismuto & zona rectal & 0,5 & 20000000 & 2 & 4,247 & 0,5309 & 0,4000 & $1 \mathrm{FTU}(1 \mathrm{FTU}=0,5 \mathrm{~g})$ \\
\hline Salisán & Salicilato de metilo & todo el cuerpo & 20,25 & 30000000 & 4 & 6,371 & 0,7964 & 0,4000 & \\
\hline Salisán & Alcanfor & todo el cuerpo & 20,25 & 30000000 & 4 & 6,371 & 0,7964 & 0,4000 & \\
\hline Clotrimazol & Clotrimazol & todo el cuerpo & 20,25 & 30000000 & 3 & 6,371 & 0,7964 & 0,4000 & \\
\hline Clobetasol & Clobetasol propionato & todo el cuerpo & 20,25 & 20000000 & 2 & 4,247 & 0,5309 & 0,4000 & \\
\hline Cortibiot gel & Hidrocortisona base & todo el cuerpo & 20,25 & 16800000 & 2 & 3,568 & 0,4460 & 0,4000 & \\
\hline Cortibiot gel & Neomicina sulfato & todo el cuerpo & 20,25 & 16800000 & 2 & 3,568 & 0,4460 & 0,4000 & \\
\hline Corticoide gel & Hidrocortisona & todo el cuerpo & 20,25 & 16000000 & 2 & 3,568 & 0,4247 & 0,4000 & \\
\hline Base hidrofilica & Base hidrofílica & todo el cuerpo & 20,25 & 31500000 & 2 & 6,690 & 0,8362 & 0,4000 & \\
\hline Crema hidratante & Crema hidratante & todo el cuerpo & 20,25 & 31500000 & 2 & 6,690 & 0,8362 & 0,4000 & \\
\hline Secavit & Vitamina A & nalgas del bebé & 3,5 & 49000000 & 8 & 10,406 & 1,3008 & 0,4000 & $1 \mathrm{FTU}(1 \mathrm{FTU}=0,5 \mathrm{~g})$ \\
\hline Ungüento vitaminado & Vitamina A & todo el cuerpo & 20,25 & 35000000 & 8 & 7,433 & 0,9291 & 0,4000 & \\
\hline Protector solar & $\begin{array}{c}\text { Benzofenona } 3 \\
\text { (Escalol 567) }\end{array}$ & todo el cuerpo & 20,25 & 49000000 & 4 & 10,406 & 1,3008 & 0,4000 & \\
\hline Protector solar & Silicato de octilo & todo el cuerpo & 20,25 & 49000000 & 4 & 10,406 & 1,3008 & 0,4000 & \\
\hline Protector solar & $\begin{array}{l}\text { p-metoxicinamato de } \\
\text { octilo (Escalol 557) }\end{array}$ & todo el cuerpo & 20,25 & 49000000 & 4 & 10,406 & 1,3008 & 0,4000 & \\
\hline Vaselina sólida & Vaselina sólida & todo el cuerpo & 20,25 & 28000000 & 2 & 5,946 & 0,7433 & 0,4000 & \\
\hline Gel alcohol $70^{\circ}$ & Gel alcohol $70^{\circ}$ & manos & 0,5 & 13000000 & 12 & 2,761 & 0,3451 & 0,4000 & $1 \mathrm{FTU}(1 \mathrm{FTU}=0,5 \mathrm{~g})$ \\
\hline Gel para imagenología & Gel para imagenología & abdomen y espalda & 3,5 & 93000000 & 1 & 19,750 & 2,4688 & 0,4000 & $7 \mathrm{FTU}(1 \mathrm{FTU}=0,5 \mathrm{~g})$ \\
\hline & & & & & & 2,7608 & 0,3451 & 0,4000 & \\
\hline & & & & & & mín L1 & min L2 & mín L3 & \\
\hline & & & & & & 2,7608 & 0,3451 & 0,4 & \\
\hline & & & & & & \multicolumn{2}{|c|}{ Límite mg/hisopado } & 0,3451 & \\
\hline & & & & \multicolumn{2}{|c|}{ Vdesorción $=5 \mathrm{~mL}$} & \multicolumn{2}{|c|}{ Límite $\mathrm{mg} / \mathrm{mL}$} & 0,0690 & \\
\hline
\end{tabular}

Figura 5. Cálculo y selección del límite de limpieza.

\begin{tabular}{|c|c|c|c|c|c|c|c|c|c|}
\hline \multicolumn{10}{|c|}{ Selección del producto crítico, A. Semisólidos. Lotes chicos. } \\
\hline Producto & Activo & Tipo & Solubilidad en agua & Valor & $\begin{array}{c}\text { LD50 Vo } \\
(\mathrm{mg} / \mathrm{kg})\end{array}$ & Descripción toxicidad & Valor & $\begin{array}{c}\text { Dificultad } \\
\text { de limpieza }\end{array}$ & $\begin{array}{l}\text { PUNTAJE } \\
\text { FINAL }\end{array}$ \\
\hline Nitroglicerina $0,2 \%$ & $\begin{array}{l}\text { Nitroglicerina } 20 \% \\
\text { solución }\end{array}$ & Ungüento & levemente soluble & 3 & 105 & muy tóxico & 3 & 3 & 9 \\
\hline Antipirina & Antipirina & Ungüento & muy soluble & 1 & 1310 & moderadamente tóxico & 2 & 3 & 6 \\
\hline Pasta de Unna & NA & Gel & NA & 0 & NA & NA & 0 & 1 & 1 \\
\hline
\end{tabular}

Figura 6. Valoración de productos semisólidos en tren de equipos para lotes chicos. 


\begin{tabular}{|c|c|c|c|c|c|c|c|c|c|}
\hline Producto & Activo & Tipo & Solubilidad en agua & Valor & $\begin{array}{c}\text { LD50 Vo } \\
(\mathbf{m g} / \mathbf{k g})\end{array}$ & Descripción toxicidad & Valor & $\begin{array}{c}\text { Dificultad } \\
\text { de limpieza }\end{array}$ & $\begin{array}{c}\text { PUNTAJE } \\
\text { FINAL }\end{array}$ \\
\hline Nitroglicerina 0,2\% & $\begin{array}{c}\text { Nitroglicerina 20\% } \\
\text { solución }\end{array}$ & Ungüento & levemente soluble & 3 & 105 & muy tóxico & 3 & 3 & 9 \\
\hline
\end{tabular}

Figura 7. Producto con mayor riesgo total.

\section{Selección del producto B y cálculo del límite de limpieza.}

\begin{tabular}{|c|c|c|c|c|c|c|c|c|c|c|c|c|}
\hline \multicolumn{13}{|c|}{ Producto A: Nitroglicerina } \\
\hline Producto $\mathrm{B}$ & Activo B & Envase & $\begin{array}{c}\text { Área de } \\
\text { aplicación }\end{array}$ & $\begin{array}{c}\text { Área de } \\
\text { aplicación }\end{array}$ & $\begin{array}{c}\mathrm{g} \\
\text { FTU/aplicación }\end{array}$ & $\begin{array}{l}\text { Lote B } \\
(\mathrm{mg})\end{array}$ & $\begin{array}{c}\text { máx } \\
\text { ap/día }\end{array}$ & $\begin{array}{l}\text { L1 }(\mathrm{mg}) \\
\text { método I }\end{array}$ & $\begin{array}{l}\text { L1 }(\mathrm{mg}) \\
\text { método II }\end{array}$ & $\begin{array}{c}\mathrm{L} 2 \\
(\mathrm{mg})\end{array}$ & L3 (mg) & $\begin{array}{c}\text { Observacio- } \\
\text { nes }\end{array}$ \\
\hline Antipirina & Antipirina & pomo & todo el cuerpo & 11583 & 20,25 & 7500000 & 3 & 0,0013 & 0,0013 & 0,3849 & 0,4000 & \\
\hline $\begin{array}{c}\text { Nitroglicerina } \\
0,2 \%\end{array}$ & $\begin{array}{l}\text { Nitroglicerina } \\
20 \% \text { solución }\end{array}$ & pomo & zona rectal & 286 & 0,5 & 8000000 & 2 & 0,0821 & 0,0821 & 0,4105 & 0,4000 & $\begin{array}{c}1 \text { FTU }(1 \text { FTU } \\
=0,5 \mathrm{~g})\end{array}$ \\
\hline & & & & & & \multirow{2}{*}{\multicolumn{2}{|c|}{ Vdesorción $=5 \mathrm{~mL}$}} & \multicolumn{2}{|c|}{ Límite $\mathrm{mg} / \mathrm{hisopado}$} & & 0,0013 & \\
\hline & & & & & & & & \multicolumn{2}{|c|}{ Límite $\mathrm{mg} / \mathrm{mL}$} & & 0,000253 & \\
\hline
\end{tabular}

Figura 8. Cálculo y selección de límite de limpieza.

\section{DISCUSIÓN}

El estudio de riesgo realizado permitió determinar dos activos críticos desde el punto de vista de la limpieza, uno para cada tren de equipos. Se determinaron también los límites de concentración máximos permitidos en superficie para dichos activos, luego de aplicar los procedimientos de limpieza correspondientes. Al considerar el límite de limpieza más estricto se tendrá la certeza de que si la superficie se encuentra libre del activo más difícil de limpiar se encontrará libre de todo activo.

Al separar en dos trenes de equipos de producción se asegura la evaluación de todos ellos.

La determinación de la unidad posológica en formas farmacéuticas de uso tópico requiere una serie de consideraciones que fueron tomadas en cuenta durante este estudio, ya que no está claramente definida por la presentación de la forma farmacéutica. La utilización de la unidad definida como FTU permitió definir la cantidad de activo en cada aplicación y así aplicar los criterios de cálculo de límites de manera representativa del uso del producto.

\section{CONCLUSIONES}

El método del peor caso aplicado a un estudio de riesgo permitió determinar los principios activos críticos empleados en la fabricación de semisólidos Se calcularon los límites de limpieza determinando la unidad posológica para semisólidos con el método de Fourman y Mullen adaptado a formas semisólidas mediante el criterio de Long y Finley de forma racional, siendo una herramienta indispensable para el cálculo de límites durante la validación de limpieza de estos productos.

\section{REFERENCIAS}

(1) Ovais M, Lian LY. Setting Cleaning Validation Acceptance Limits for Topical Formulations.

Pharm Technol 2008; 32(1):96.

(2) Fourman GL, Mullen MV. Determining Cleaning Validation Acceptance Limits for Pharmaceutical Manufacturing Operations.

Pharm Technol 1993; 17(4):54.

(3) Oakley A. Fingertip Unit. Disponible en: https:// www.dermnetnz.org/topics/fingertip-unit. [Consulta 13/06/2018] Sitio web. 1 pagina en pantalla. 